\title{
In Situ Micropillar Deformation of Hydrides in Zircaloy-4
}

\author{
H. E. Weekes ${ }^{\mathrm{a}}$, V. A. Vorontsov ${ }^{\mathrm{a}}$, I.P. Dolbnya ${ }^{\mathrm{b}}$, J. D. Plummer ${ }^{\mathrm{a}}$, F. Giuliani ${ }^{\mathrm{a}}$, \\ T. B. Britton ${ }^{\mathrm{a}}$, D. Dye $\mathrm{D}^{\mathrm{a}, *}$ \\ ${ }^{a}$ Department of Materials, Royal School of Mines, Imperial College London, Prince Consort \\ Road, London, SW7 2BP, UK \\ ${ }^{b}$ Diamond Light Source, Harwell Science and Innovation Campus, Didcot OX11 ODE, UK
}

\begin{abstract}
Deformation of hydrided Zircaloy-4 has been examined using in situ loading of hydrided micropillars in the scanning electron microscope and using synchrotron $\mathrm{X}$-ray Laue microbeam diffraction. Results suggest that both the matrix and hydride can co-deform, with storage of deformation defects observed within the hydrides, which were twinned. Hydrides placed at the plane of maximum shear stress showed deformation within the hydride packet, whilst packets in other pillars arrested the propagation of shear bands. X-ray Laue peak broadening, prior to deformation, was associated with the precipitation of hydrides, and during deformation plastic rotation and broadening of both the matrix and hydride peaks was observed. Post-mortem TEM of the deformed pillars has indicated a greater density of dislocations associated with the precipitated hydride packets, while the observed broadening of the hydride electron diffraction spots further suggests that plastic strain gradients were induced in the hydrides by compression.
\end{abstract}

Keywords: zirconium, synchrotron diffraction, TEM, micromechanics, hydrides

\section{Introduction}

Zirconium alloys are widely used as nuclear fuel cladding material and readily absorb hydrogen during service in a pressurized water reactor (PWR) primary circuit environment. Possible mechanisms allowing hydrogen to be either generated in or introduced into the clad material include corrosion, radiolysis of water, fuel oxidation and dissolved coolant hydrogen [1]. Although able to accept up to 50 at.\% hydrogen in solid solution at temperatures above $500^{\circ} \mathrm{C}$, as temperature decreases, so does the alloy's ability to accommodate hydrogen in

\footnotetext{
* Corresponding author.

Email address: david.dye@imperial.ac.uk (D. Dye)
} 
solution. At room temperature, the solubility may be as low as $10^{-4}$ at.\% [2]. This leads to the formation of embrittling zirconium hydrides, particularly during cooling from the operating temperature $\left(\sim 350^{\circ} \mathrm{C}\right)$.

Hydride formation is generally considered detrimental to mechanical properties and results in decreases in fracture strength, impact strength and tensile ductility at slow strain rates [2]. The extent of hydride embrittlement on the accommodating $\mathrm{Zr}$ matrix is found to be critically dependent on both their morphology and orientation relative to the applied load direction [2]. Although the texture of zirconium alloys can be controlled so as to promote hydride precipitation in relatively benign orientations, hydrides can reorient to undesirable orientations (perpendicular to load direction) during cooling under an applied tensile stress [3-5]. Enhanced hydride nucleation is readily observed within the highly stressed region of a crack tip and, together with hydride reorientation under a tensile stress, results in the initiation of the intermittent crack growth process known as delayed hydride cracking (DHC). DHC was initially not considered a major issue at temperatures below $200^{\circ} \mathrm{C}$ [6], but reports of component failures after long term room temperature storage [7] have led to greater attention to this phenomenon.

Individual zirconium hydrides display a characteristic acicular, or plate-like, morphology with a habit plane parallel to $\{0002\}_{\mathrm{Zr}}$. A $\{10 \overline{1} 7\}_{\mathrm{Zr}}$ habit plane is found for the macroscopic hydride packets observed by optical microscopy, which was studied by transmission electron microscopy (TEM) by Chung et al. [8], Figure 1(a). The plate size and morphology is a consequence of transformation strains, and have been rationalised with the phenomenological theory of martensite transformations [9-12]. Individual nano-hydride platelets are autocatalytically nucleated in macroscopic hydride packets, several $\mu \mathrm{m}$ in length, via a process of strain accommodation. The sharp tips of these nano-hydrides have been observed to punch dislocation loops into the surrounding $\mathrm{Zr}$ matrix $[13,14]$, illustrated in Figure 1(b) ${ }^{1}$. These shear loops should be distinguished from the prismatic loops that can be observed due to irradiation. It should also be noted that the dislocations observed around surface hydrides generated by electropolishing or around hydrides induced by quenching may not be representative of the state where hydrides form on slow cooling.

[Figure 1 about here.]

In recent years, the field of experimental small scale micromechanics has grown dramatically. Demonstration experiments performed on the deformation of pillars, $1-10 \mu \mathrm{m}$ in width, have highlighted the potential of $i n$ situ synchrotron $\mathrm{X}$-ray Laue microbeams to probe the deformation response of materials [1517], although only ideal or 'model' materials have been examined. Changes in lattice structures, such as rotation gradients and sub-grain formation, have

\footnotetext{
${ }^{1}$ The reader should note that there is a concern in the community as whether the hydrides studied in Carpenter et al. [14] were artefacts introduced by polishing. In addition, Carpenter et al. studied $\gamma$ hydrides, not $\delta$ - $\mathrm{ZrH}$
} 
been observed through diffraction peak evolution. The sensitivity of microbeam diffraction to defect structures has also enabled the study of dislocation density evolution through both the streaking and broadening of diffraction peaks. It is therefore timely to ask if such experiments can provide useful insights into the deformation response of real, industrial, micromechanics problems such as that of hydrides in zirconium.

In the present work, we examine the deformation behaviour of electrolytically hydrided Zircaloy-4 Focussed Ion Beam (FIB) milled micropillars. Loaded in compression and oriented at approximately $45^{\circ}$ to the loading axis, the hydride plates were positioned near to the plane of maximum shear. First, in situ testing in within a Scanning Electron Microscope (SEM) chamber is presented in order to elucidate the general deformation behaviour of the pillars. Then, the evolution of the diffraction patterns obtained by in situ microbeam synchrotron Laue diffraction is examined. Finally, the post-loading dislocation states are explored via (Scanning) Transmission Electron Microscopy, (S)TEM, using thin foils FIB-milled from the deformed pillars.

\section{Experimental}

Electrolytic hydriding was carried out in dilute sulphuric acid $\left(1 \% \mathrm{H}_{2} \mathrm{SO}_{4}\right)$ at $65^{\circ} \mathrm{C}\left( \pm 5^{\circ} \mathrm{C}\right)$ using a current density of $2 \mathrm{kAm}^{-2}$. Heat treatment then interdiffused the hydrogen uniformly through the sample and a slow furnace cool promoted the formation of stable $\delta$ hydrides. The $\mathrm{Zr}$ texture acquired through hot rolling aligns the basal poles perpendicular to the rolling direction $\left( \pm 20^{\circ}\right)[18]$ and Figure 1(c) highlights the resulting hydride alignment due to the known $(0002)_{\mathrm{Zr}} \|(111)_{\mathrm{ZrH}}$ orientation relationship. Hydride precipitation readily occurs at $\alpha$ - Zr grain boundaries, and to avoid this the sample was annealed at $830^{\circ} \mathrm{C}$ for $24 \mathrm{~h}$ to promote both grain growth and precipitation of intragranular hydrides. Figure 1(d) shows the microstructure and hydrides after the applied heat treatment.

A grain of interest with its $\{0002\}$ close to $45^{\circ}$ from the sample surface was identified using electron backscatter diffraction (EBSD) and, for pillars to be used during in situ diffraction experiments, sliced to produce a thin wedge with a $50 \mu \mathrm{m}$ platform edge for fabrication. In situ SEM pillars were produced directly from the bulk. Slice and view FIB milling, Figure 2(a), coupled with sample surface EBSD analysis located hydrides oriented near $45^{\circ}$ from the loading axis. Perpendicular focussed ion beam (FIB) milling (30keV with a final polishing step of 30pA), was then used to produce pillars with a slight taper and aspect ratio of $\sim 3: 1$. In this geometry, one end of the pillar remained part of the bulk while the other was a free surface. All the FIB milling presented here was performed using an FEI Helios Nanolab600 FIB-SEM.

[Figure 2 about here.]

In situ SEM micro-compression experiments were performed using an Alemnis SEM indenter placed in a Zeiss Auriga FIB-SEM, Figure 2(b). The indenter 
stage was oriented at $30^{\circ}$ to the electron beam, ensuring adequate visibility of the pillar sides. A single crystal diamond flat punch tip with a $10 \mu \mathrm{m}$ end diameter was used as the compression anvil.

Microbeam X-ray Laue diffraction experiments were carried out using a custom-built nano-indentation loading rig utilising a Hysitron indenter at beamline B16 at Diamond Light Source, Figure 2(c). Preliminary experiments, not shown here, also used the facilities of ALS (Advanced Light Source) and APS (Advanced Photon Source). The fabricated pillars were located and positioned beneath a flat punch indenter using a top mounted light microscope, with the compression axis perpendicular to the incident beam. A 3-axis stage allowed for accurate positioning. Once located and centred beneath the $10 \mu \mathrm{m}$ diameter diamond indenter, the pillars were exposed to a polychromatic X-ray beam, with an energy range of 4-24 keV, in a transmission geometry. Kirkpatrick-Baez mirrors were used to focus the X-ray beam to produce a beam of 0.8-1 $\mu \mathrm{m}$ (FWHM). A large area (3056 x 3056 pixel, $31 \mu \mathrm{m}$ pixel size) CCD detector (ImageStar 9000 area detector, Photonic Science Ltd) at a sample-detector distance of $\sim 100$ $\mathrm{mm}$ was used to record a Laue pattern every $40 \mathrm{~s}$, with an exposure time of $1 \mathrm{~s}$. All the results presented here were obtained from load-controlled testing carried out at a loading rate of $10 \mu \mathrm{N} \mathrm{s}^{-1}$, which for the $3 \times 3 \mu \mathrm{m}$ square pillars used for the X-ray experiments corresponds approximately to $1.1 \mathrm{MPa} \mathrm{s}^{-1}$ at the top of the tapered pillar.

Due to the limited number and distorted nature of the generated Laue spots, lattice orientation determination via collective spot indexing directly from the Laue patterns was not possible. Instead, the crystal orientation of the pillars was established using EBSD. A $36 \times 29 \mu \mathrm{m}$ map with a $0.1 \mu \mathrm{m}$ step size was produced. The expected Laue diffraction patterns were then simulated to verify the orientation. After compression, lattice rotation was investigated using EBSD of the front facing pillar surfaces. Low current $(30 \mathrm{pA})$ parallel FIB-milling was used to produce the flat, polished surfaces required.

In order to gain insight into the operative deformation mechanisms, deformed micropillars were examined by site-specific TEM. During preparation of the TEM foils, the pillars were tilted at $52^{\circ}$ to place their surface normals parallel to the $\mathrm{Ga}^{+}$beam. Pt was deposited to cover the pillar surface before trenches were FIB-milled either side at $30 \mathrm{keV}$ and $21 \mathrm{nA}$. Once thinned, the foil was lifted out using a microprobe and attached, via Pt-deposition, to a $\mathrm{Cu}$ grid. Further polishing at varying tilts around $52^{\circ}$, at progressively lower accelerating voltages (down to $2 \mathrm{keV}$ ), attained an electron transparent section suitable for TEM analysis.

\section{Results}

\subsection{In situ SEM microcompression}

In situ SEM micropillar compression tests were conducted on as-received Zircaloy-4 (pillar AR1) and two hydride containing Zircaloy-4 pillars (H1 and H2). All three pillars were fabricated from different grains of the same sample. 
The sample, having undergone an annealing heat treatment to increase its grain size, contained multiple intragranular macro hydride packets.

The load-displacement curves are shown in Figure 3, and include a secondary nominal stress axis. The conversion has been made using the measured mid-height sample cross-sectional area and nominal length, based on SEM measurements; it should be appreciated that such conversions are approximate. During the compression of the unhydrided sample AR1 (Figure 3a) linear elastic loading was observed up to $\sim 530 \mathrm{MPa}$, where macroscopic yielding occurred. This strength is greater than that for the bulk alloy, which has a strength of $370-460 \mathrm{MPa}[19]$.

[Figure 3 about here.]

Figure 4 shows the sample surface appearance at points (a)-(c) highlighted during the loading curve in Figure 3(a). Deformation initiated at the top corner of the pillar, Figure $4 \mathrm{~b}$, and produced a shear band (1) inclined $\sim 40^{\circ}$ from the loading axis. As deformation proceeded, a secondary shear band (2) formed, perpendicular to (1) and approximately $50^{\circ}$ to the loading axis. Investigation of the crystallographic rotations that occurred during loading, due to the effect of constraint from the platen and built-in end, was then carried out using EBSD. FIB milling was used to remove the pillar from the substrate and to polish a flat surface for EBSD examination, in the same manner as that used for TEM preparation. Shear band (2) in Figure 4(c) resulted in a rotation about the $\{0001\}$ axis, indicative of $<\mathrm{a}>$ slip in the $\mathrm{Zr}$ matrix, Figure 5 .

[Figure 4 about here.]

[Figure 5 about here.]

Loading curves for the hydrided pillars $\mathrm{H} 1$ and $\mathrm{H} 2$ are shown in Figure 3(b-c). Both yielded at greater loads than the as-received material; 1600 and $900 \mathrm{MPa}$ compared to $530 \mathrm{MPa}$. Sample H1 also displayed significantly greater work hardening; the test of the second hydrided sample $\mathrm{H} 2$ was halted shortly after yield in order to attain a smaller amount of deformation for subsequent TEM analysis.

SEM images of hydrided sample H1 during testing are shown in Figure 6. Two distinct hydride packets were observed, (1) and (2) in Figure 6(d). EBSD performed on the top surface of the sample prior to machining of the pillars indicated that these hydrides were in twin-related orientations. At the point of yield, the formation of shear bands towards the top of the pillar was observed, indicated by the red circle in (b). These slip traces appeared to arrest at the adjacent hydride packet, without penetrating into the material below. Instead, at (c), a second shear band appeared, near the twin hydride - matrix interface.

[Figure 6 about here.]

The primary shear band is intersected by the parent hydride packet and postmortem analysis has indicated plastic deformation within the hydride packet 
itself. Figure 6(e-f) presents evidence of slip traces spanning the width of the hydride packet sitting across the pillar. This is consistent with the occurrence of slip between the individual microscopic hydride platelets that stack up to form the larger, and more readily observed, macroscopic packets. EBSD carried out on pillar $\mathrm{H} 1$ prior to loading established that the (0002) plane normal was oriented $\sim 30^{\circ}$ from the pillar (loading) axis. This is consistent with the angle of the observed shear bands suggesting shearing consistent of rigid body displacement of the hydride platelets (where $\mathrm{ZrH}_{\{111\}} \| \mathrm{Zr}_{\{0002\}}$ ). The association between the macro-hydride plates and micro-hydride platelets is often considered in terms of the individual stacking of platelets along the $\{10 \overline{1} 7\}$, consequently providing an apparent near basal trace. However, the nature of the hydrides observed after compression in $\mathrm{H} 1$ suggests a different stacking orientation across the macroscopic packet.

SEM images of H2 during compression are shown in Figure 7 where $(\mathrm{a}-\mathrm{c})$ correspond to $(\mathrm{a}-\mathrm{c})$ in Figure 3c. Macroscopic hydride plates oriented $\sim 40^{\circ}$ to the loading axis are visible in Figure $7 \mathrm{~d}$, outlined. As loading proceeded, strain localisation was initially observed along, or close to, the hydride-matrix ( $\mathrm{ZrH}-$ $\mathrm{Zr}$ ) interface, Figure 7b. Further loading resulted in deviation away from this initial slip path and into the neighbouring $\mathrm{Zr}$ matrix. However, this was unable to be maintained and slip reinitiated along the $\mathrm{ZrH}-\mathrm{Zr}$ interface at the base of the pillar. The corresponding points along the stress-strain curve indicated a small strain burst at (b), where initial deformation along the $\mathrm{ZrH}-\mathrm{Zr}$ interface was observed (Figure 7b). A small region of strain hardening, potentially stemming from the slip path deviation and retardation in the adjacent matrix, was observed prior to macroscopic shearing at (c), a strain of $\sim 7 \%$.

[Figure 7 about here.]

After compression the second hydride-containing pillar, H2, was removed from the bulk and FIB-polished to produce the flat surface required for EBSD analysis. Figure 8 summarises the data obtained. Two distinct hydride orientations were observed (1) and (2) within the EBSD map (b) and in the corresponding misorientation profile $\left(\mathrm{c}_{1}\right)$ the boundary was found to have a misorientation of $\sim 25^{\circ}$ (referring to the starting point of the line scan). A continuous change in orientation was observed along hydride packet $(2), c_{2}$ and $c_{3}$.

[Figure 8 about here.]

\subsection{In situ x-ray Laue diffraction microcompression}

\subsubsection{Mechanical behaviour}

In situ 'pink' microbeam Laue diffraction experiments were carried out during the compression of an unhydrided Zircaloy-4 pillar (ARL1) and two hydridecontaining Zircaloy-4 pillars (HL1 and HL2). The load-displacement curves are shown in Figure 9. Pillars were fabricated from a single large grain with its $c$-axis $\sim 40^{\circ}$ to the loading axis; however, the loading response was strongly dependent on the microstructure of the pillar. 
[Figure 9 about here.]

During the loading of the unhydrided pillar ARL1, Figure 9, linear elastic loading was observed until a strain of $\sim 1.5 \%$ was reached, at a load of $\sim$ $3.6 \mathrm{mN}$. Beyond this point, intermittent plastic strain bursts were observed. The plateau corresponds to the $180 \mathrm{~s}$ load hold that was imposed in each case prior to unloading. Figure 10 $\left(\mathrm{a}_{1}\right)-\left(\mathrm{a}_{2}\right)$ compares SEM images of ARL1 before (a) and after (b) compression. Very little $z$-axis rotation (about the beam) was observed, and in addition several slip traces were observed towards the base of the pillar.

[Figure 10 about here.]

Figure 9 also presents the load-displacement data for HL1. The point of yield cannot be clearly identified. Once plasticity had begun, as with ARL1, intermittent strain bursts were observed. Figure 10 compares SEM images of HL1 before $\left(b_{1}\right)$ and after $\left(b_{2}\right)$ compression and shows distinct deformation predominantly in the bottom and mid-sections of the pillar. The majority of the slip traces observed were remote from the major hydride packet towards the top of the pillar, but their plane was similar in orientation to that of the packet. In addition, the rotation of the pillar was more apparent towards the top of the pillar, in the region of the packet.

For hydrided pillar HL2, linear elastic loading was observed until approximately $600 \mathrm{MPa}$. In this sample, homogeneous and continuous but limited strain hardening was observed until the load hold. Before-and-after micrographs of the pillar, Figure $10\left(c_{1-2}\right)$ show that a distinct slip band was produced near the plane of maximum shear, close to the $\{0002\}_{\mathrm{Zr}}$ and just below the hydride packet.

\subsubsection{Diffraction data}

The intensity distribution of a Laue diffraction spot, from a well focused beam, is a consequence of (a) the energy distribution and divergence of the incident beam, (b) the sampling of the illuminated object by the beam, and (c) microstructure within the object. Therefore, for a sample larger than the beam with uniform crystal orientation and lattice spacings, a near-perfect, circular diffraction spot will be obtained. Deviations of plane spacing and orientation gradients will introduce structure into the Laue spot [20,21].

Within a diffraction pattern, the structure of the spots from a material containing defects is a consequence of the strain fields and lattice curvatures present within the illuminated volume. In some special cases these can be uniquely determined, but in many others, these are non-unique and hypotheses must be tested; such hypotheses may be found to be consistent with the observed spot structure.

Initial peak shape. The crystal orientation of the pillars was established using EBSD, at a location just below the linear array of pillars. These experimentally observed Laue reflections were then indexed by manual comparison 
with simulated patterns generated using the CrystalDiffract software. Using the $h c p\left(\mathrm{P} 6_{3} / \mathrm{mmc}\right)$ crystal structure with lattice parameters $a=3.2276 \AA$ and $c=5.1516 \AA$ produced the Laue pattern simulated in Figure 11(b), allowing direct comparison to the experimental data, Figure 11(a). Figure 11b is colour coded with respect to diffraction peak intensity and the distinct, high intensity, reflections highlighted correspond to the experimentally observed reflections $(\overline{1} 2 \overline{1} 0)$ and $(2 \overline{1} \overline{1} 0)$.

[Figure 11 about here.]

The intensity distribution of each Laue spot observed in hydride containing pillars HL1 and HL2 prior to loading was highly asymmetric, with streaking occurring predominantly away from the transmited beam, with an intense maximum at the centre of each streak. Figure $12(\mathrm{a}-\mathrm{c})$ compares the $(2 \overline{1} \overline{1} 0)$ reflections of ARL1, HL1 and HL2. Notably, AR1 has a far more symmetric peak than the hydride-containing pillars prior to deformation. Multiple small scale testing experiments have recorded peak broadening prior to deformation [22-24], and this has been attributed to the presence of deviatoric elastic strain or orientation gradients within the probed volume [25]. Investigation into the effect of sample preparation on initial microstructure has indicated a distinct correlation between FIB milling and the presence of structural defects, for example high dislocation densities $[26,27]$. This leads to the assumption that the asymmetry of the initial reflections prior to loading for ARL1 is an artefact of the pillar preparation process. In contrast, the much enhanced streaking of HL1 and HL2 must be a consequence of the presence of the hydride phase.

[Figure 12 about here.]

Figure 12(d-f) illustrates a hypothesis as to the origin of this enhanced streaking in the hydride-containing pillars. Considering HL1 first (b and e), two distinct maxima can be observed, (I) the principal Zr peak and a second, much fainter peak (II), which is at a different radial position. This can be described by the $f c c(\mathrm{Fm} \overline{3} \mathrm{~m}) \delta-\mathrm{ZrH}_{1.66}$ phase, with $a=4.768 \AA$. If the beam was primarily encompassing the $\mathrm{Zr}$ phase, with a small portion of the hydride phase, a diffraction pattern of this nature is expected. Moving on to consider HL2 (c and f), a third maximum was observed. This can consistently be described by a second $h c p$ Zr orientation, with the same lattice parameters. This is depicted in (f), illustrating the situation where the top and bottom of the pillar are in slightly different orientations, separated by a hydride packet. In addition to the multiple peak maxima, extensive peak streaking is also observed in HL2, with reduced streaking also shown in HL1. This is considered to be due to the presence of induced coherency strain gradients within the matrix surrounding the precipitated hydride [13].

Using the orientations of the $\alpha$-Zr and $\delta$-hydride established by EBSD, the combined Laue pattern for both phases was generated, Figure 13(a). Thus, the $\delta$-ZrH $(2 \overline{2} 0)$ peak appears just inside the $\alpha$-Zr $(2 \overline{1} \overline{1} 0)$ peak, as observed in Figure 12(b). This is constant with the known orientation relationship, 
$(0002)_{\alpha} \|(111)_{\delta}$. An elastic strain, changing the $c / a$ ratio, as examined in Figure 13(b), does not generate motion of the $(2 \overline{1} \overline{1} 0) \mathrm{Zr}$ peak, and therefore cannot be attributed to Zr peak (III) in Figure 12(c).

[Figure 13 about here.]

The effect of crystal rotations and shears on the expected diffraction patterns is shown in Figure 14. All of these produce radial rotations on the detector, with the exception of rotation of the crystal about the beam $(z)$ axis, which only produces azimuthal rotation. Therefore it can be stated clearly that crystal rotations and/or shears can account for the observation of the secondary $\alpha$ $\mathrm{Zr}$ maximum (III) in Figure 12(c). The three maxima observed cannot be explained by a continuous, homogeneous deformation around the hydride, but are consistent with the depiction in Figure 12(f).

[Figure 14 about here.]

Evolution during compression. During loading, diffraction patterns were recorded every 40 seconds. Figure 15 (b) shows the evolution of the $(2 \overline{1} \overline{1} 0)$ peak during loading of the unhydrided pillar AL1. The initially well-defined Laue peak $\left(b_{1}\right)$ first began to broaden and rotate $\left(b_{2}\right)$. As it was loaded, it rotated to a smaller diffraction angle, indicative of both deviatoric elastic loading and plasticity. As load was increased further, a number of subsidiary maxima appeared $\left(b_{3}\right)$, which were adjacent to the original diffraction peak. At the end of loading, extensive streaking away from the transmitted beam was observed, coupled with peak splitting of the sub-peak perpendicular to the dominant axis. These peak characteristics remained on unloading. Post mortem analysis of AL1 through electron microscopy $\left(a_{1}\right)$ and EBSD mapping of the deformed surface $\left(a_{2}, c\right)$ revealed the presence of an extension $\{10 \overline{1} 2\}$ twin at the base of the pillar, characterised by its $85^{\circ}$ misorientation around $\{10 \overline{1} 2\}$ [28]. Interestingly, the streaking away from the transmitted beam with subsidiary maxima may be in part due to this twin, with the extra peak corresponding to the $(12 \overline{3} 4)$ peak from the twin.

[Figure 15 about here.]

Figure 16 shows the evolution of the primary diffraction peaks observed in hydride-containing pillars HL1 and HL2 during loading. For HL1, both the $(2 \overline{1} \overline{1} 0)$ and $(\overline{1} 2 \overline{1} 0)$ reflections were visible. Notably, in HL1, the initial $(2 \overline{1} \overline{1} 0)$ peak was spread over a much wider range of diffraction angles, and a faint satellite peak could be discerned. Beyond a load of $190 \mathrm{MPa}$, Figure $16\left(\mathrm{~b}_{2}\right)$, a steady increase in azimuthal width of the primary peak could be observed. By $750 \mathrm{MPa}$, considerable peak splitting was observed, accompanied by an increase in intensity of the satellite peak. Similar peak splitting is observed for the $(\overline{1} 2 \overline{1} 0)$ reflection. This rotation is consistent with rotation about the $\mathrm{X}$ and $\mathrm{Y}$ axes (Figure 14(a)(b)), in different senses, by the material immediately below the hydride packet. On unloading, some of the splitting of the primary peak was 
observed to relax away. The $(2 \overline{2} 0)_{\delta}$ peak observed in Figure 16 shows minimal change throughout loading, with only a slight increase in radial streaking. Its increase in intensity can be attributed to motion of the pillar relative to the beam as the pillar is compressed.

[Figure 16 about here.]

For HL2, only the $(2 \overline{1} \overline{1} 0)$ peak was observed, and its evolution through loading is considered in terms of its three component peaks (I), (II) and (III), highlighted in Figure 16( $\left.\mathrm{c}_{1}\right)$. At the beginning of loading, minimal peak broadening was observed. An increase in (I) intensity was observed as (III) intensity reduced at a load of approximately $400 \mathrm{MPa}(\mathrm{c} 3)$. This is indicative of motion of the beam location relative to the pillar height as it is compressed. At $650 \mathrm{MPa}$, subsidiary peaks from the hydride (II) and top part of the pillar (III) began to rotate away, leaving the bottom portion of the pillar (I) undisturbed. After unloading, much of this peak rotation again relaxed back towards the initial configuration, leaving behind a long, faint diffraction streak.

In addition, an increase in streaking towards/away from the transmitted beam was observed in the $(2 \overline{1} \overline{1} 0)$ peak for both HL1 and HL2 during loading. Weak in their intensity relative to the primary peak, this is considered to be a result of strain gradient accumulation due to the presence of geometrically necessary dislocations (GNDs), with a smooth orientation gradient [29, 30], in the presence of constraint due to the platens and built-in end.

SEM macrographs of HL1 and HL2 after loading, Figure $10\left(b_{2}\right)$ and $\left(c_{2}\right)$, confirm the rotations observed via diffraction. In HL1, Figure $10\left(\mathrm{~b}_{2}\right)$ suggests that rotation occurred around the hydride packet, consistent with the minimal movement of the $(2 \overline{2} 0)$ hydride peak. $z$-axis rotation was also evident after compression yet this was not observed in the peak behaviour during the majority of loading. Therefore, it can be inferred that this rotation occurred at the end of loading and/or only at the very top of the pillar, above of the diffracting volume. For HL2, Figure $10\left(\mathrm{c}_{2}\right)$ shows motion of the the hydride packet and the material above. This is consistent with the observed diffraction peak behaviour where a lone intense spot remained in the original position (representative of the undeformed region at the base of the pillar) with the generated sub-peak representing deformation of the pillar top.

\section{3. $T E M$}

TEM foils were removed by FIB milling in order to investigate deformation mechanisms. Figure 17 considers hydride-containing pillar $\mathrm{H} 1$ after compression and displays high levels of deformation throughout. Dark-field imaging using a cubic hydride peak was not able to fully resolve individual hydride platelets but their characteristic stacking nature can be clearly identified (c)(d). Regions of increased hydride density, illuminated in (d) and selectively outlined in red in (c)(d), appear to show extensive strain contrast, possibly associated with dislocation build-up. When compared to regions of negligible hydride density, outlined in white in $(\mathrm{c})(\mathrm{d})$, a reduction in apparent deformation is observed. 
Asymmetric broadening of the hydride diffraction spots, circled in Figure 17(b), is visible for pillar $\mathrm{H} 1$ and is consistent with strain contrast observed in Figure $17(\mathrm{c})(\mathrm{d})$.

[Figure 17 about here.]

TEM analysis from deformed hydride-containing pillar H2 is shown in Figure 18. Individual hydride platelets can be distinguished, in addition to two hydride packets of different orientations, (1) and (2) in Figure 18(b), being revealed. Dislocations are more distinguishable within the hydride platelets for H2 than for H1, Figure 18(c)(d), and appear localised at both the packetpacket interface and within the comprising platelets. This is consistent with the strain contrast observed in H1. Considering the two differently oriented hydride packets, it has been established that (1) is oriented close to the [112] zone axis whereas (2) is close to the [110]. The high resolution lattice image in Figure 18(e) shows that there is no visible zirconium matrix between the hydride packets. From Figure 18(f) it was found that the interface was formed by $(15 \overline{3})_{1} / /(\overline{2} \overline{4} 4)_{2}$.

[Figure 18 about here.]

The high indices of the interface planes suggest a high degree of incoherence, and may serve as a source of dislocation generation. Similarly to H1, streaking of the diffraction spots for both hydride packets was also observed and selected spots are circled in Figure 18(f). Diffraction spots from both hydride packets have been further magnified and display varying amounts of streaking, an indication that the separate packets have undergone different degrees of deformation. The observations from both hydride-containing pillars, in their compressed state, have therefore indicated that deformation within the hydride packets may be possible.

Analysis of a further foil, removed from hydride-containing pillar HL1, has shown internal twinning of the hydride platelets, highlighting an additional mode of deformation possible within packets, Figure 19. Figure 19(b) overlays the diffraction patterns from both parent and twin hydrides and Figure 19(d) presents a high resolution image of the parent/twin interface displaying the $(\overline{1} \overline{1} 1)_{\mathrm{P}} / /(\overline{1} 1 \overline{1})_{\mathrm{T}}$ orientation relationship. Multiple twins were observed across the pillar, all displaying the same parent/twin relationship.

[Figure 19 about here.]

\section{Discussion}

Hydrides have generally been considered as non-shearable precipitates, with documented fracture toughness values being characteristic of extremely brittle materials at both room temperature and $300^{\circ} \mathrm{C}$ [31]. However, under compressive loading, a degree of hydride ductility above $100^{\circ} \mathrm{C}$ has been observed [32-34]. A fundamental question has been whether the hydrides themselves are 
able to be penetrated by dislocations or whether they are truly non-shearable. Here, the behaviour of $\mathrm{Zr}$ micropillars containing intragranular hydride packets was examined.

During the in situ diffraction experiments, the non-hydrided material produced relatively coherent, low mosaicity diffraction spots, whereas the hydride containing pillars were found to produce much broader spots. Whilst FIB damage may result in peak broadening [24], this is unlikely to explain the difference between the non-hydride and hydride-containing pillars observed here. We have been able to attribute the subsidiary maxima to the presence of the hydrides in the packet and to rotation of the orientation of the matrix above/below the hydride packet. Since the precipitation of a hydride involves biaxial straining of the $\mathrm{Zr}$ matrix at the hydride-matrix interface $[35,36]$ (with interfacial strains of $7.2 \%$ along $[0001]_{\alpha}$ and $4.6 \%$ along $[11 \overline{2} 0]_{\alpha}$ being predicted $[36]$ ), there is likely to be accompanying plastic deformation of the matrix. Due to constraint of the matrix, a consequence of the associated volume expansion, this deformation may result in local lattice reorientation due to an excess of dislocations resulting in broader matrix diffraction spots. There may also be an effect of size broadening, particularly for the hydride peaks.

For the hydride containing pillars, both in situ SEM and diffraction experiments showed greater plastic deformation of the accommodating matrix than the individual hydride platelets and the macro hydride packets. In the SEM, shear bands were observed to initiate in the matrix and arrested at the hydride packet. Also, deformation within the plane of a packet could be observed. In situ X-ray Laue diffraction showed much more peak broadening and rotation of the matrix $(2 \overline{1} \overline{1} 0) \mathrm{Zr}$ peak phase than the hydride $(2 \overline{2} 0)$, but broadening of the hydride $(2 \overline{2} 0)$ was also observed, indicative of strain gradients within the hydride platelets themselves. This behaviour is consistent with TEM observations which showed the presence of defects within the hydride platelets. Asymmetric broadening of diffraction spots in Figures 17 and 18 and also suggested induced plastic strain gradients in the hydride phase, consistent with the microbeam Laue observations.

It therefore appears that hydrides can be sheared when present in zirconium, at least in the extreme case of micropillar deformation examined here. Their effect on the plastic behaviour of zirconium appears to increase both its strength and extent of deformation anisotropy. That is, shear within the plane of the microscopic platelets (between the platelets) may be possible yet the overall macroscopic packets can themselves act as barriers against further propagation of shear bands initiated in the adjacent matrix. This can cause individual grains to become vulnerable to strain localisation, reducing the overall ductility of the material. The detrimental effect of the hydrides, both macroscopic packets and microscopic platelets, can therefore be considered as being largely due to the morphology with which they form from the matrix. 


\section{Conclusions}

The deformation response of Zircaloy-4 micropillars containing hydride packets placed near the plane of maximum shear has been investigated in situ using SEM combined with synchrotron X-ray Laue microbeam diffraction and postmortem TEM analysis. The following conclusions can be drawn.

1. Shear bands initiated in the matrix were observed to arrest at the hydride packet, whilst in other pillars slip bands within a packet could be observed. These indicate that hydride packets are both strong out-of-plane but can deform in-plane (between the hydride platelets).

2. A greater spread of orientations and strains could be observed in the Laue diffraction patterns obtained from hydride containing pillars than from the matrix material.

3. Twinning and shear bands could be observed in pillars comprised of matrix material.

4. Hydride-containing pillars of the same orientation showed plastic rotation in situ during deformation of the hydride packet and the material above, which showed to partially relax upon unloading.

5. TEM examination of the deformed pillars has suggested that the hydrides, along with the matrix, can deform, with a greater density of dislocations being observed within and around the hydride packets. Internal twinning of the hydrides was also observed.

\section{Acknowledgements}

DD, VAV, TJP and HEW acknowledge funding from EPSRC (EP/L0025213/1, $\mathrm{EP} / \mathrm{H} 004882 / 1)$, and from Rolls Royce plc, who supplied the material used in this study; we also acknowledge helpful discussions with Ted Darby. This research used resources (34-ID-E, John Tischler) of the Advanced Photon Source, a U.S. Department of Energy (DOE) Office of Science User Facility operated for the DOE Office of Science by Argonne National Laboratory under Contract No. DE-AC02-06CH11357. We received beamtime at the Advanced Light Source (12.3.2), supported by the Director, Office of Science, Office of Basic Energy Sciences, of the U.S. Department of Energy under Contract No. DE-AC02-05CH11231. We thank Diamond Light Source for access to beamline B16 (MT8179) that contributed to the results presented here, and Andrew Malandain for technical support.

\section{Appendix: Supplementary online information}

Videos are provided online of the in situ SEM pillar compression tests. The hydride-free pillar AR1 depicted in Figure 4 is shown in Figure S1, and hydridecontaining pillars H1 (Figure 6) and H2 (Figure 7) are shown in Figures S2 and S3, respectively. 


\section{References}

\section{References}

[1] J. C. Clayton, Internal Hydriding in Irradiated Defected Zircaloy Fuel Rods, Zirconium in the Nuclear Industry: Eigth International Symposium, ASTM STP 1023, L. F. P. Van Swam, C. M. Eucken, Eds., American Society for Testing and Materials (1989) 266-288.

[2] C. E. Ells, Hydride precipitates in zirconium alloys (A review), J. Nuc. Mater. 28:2 (1968) 129-151.

[3] K. B. Colas, A. T. Motta, M. R. Daymond, M. Kerr. Hydride Platelet Reorientation in Zircaloy Studied with Synchrotron Radiation Diffraction, J. ASTM. Int. 8:1 (2011)

[4] K. B. Colas, A. T. Motta, J. D. Almer, M. R. Daymond, M. Kerr, In situ study of hydride precipitation kinetics and re-orientation in Zircaloy using synchrotron radiation, Acta Mater. 58 (2010) 6575-6583.

[5] V. Alvarez, J. R. Santisteban, P. Vizcaino, A. V. Flores, Hydride reorientation in $\mathrm{Zr}-2.5 \mathrm{Nb}$ studied by synchrotron X-ray diffraction, Acta Mater. 60:20 (2012) 6892-6906.

[6] International Atomic Energy Agency. Long Term Storage of Spent Nuclear Fuel - Survey and Recommendations: Final Report of a Co-ordinated Research Project 1904-1997 (2002).

[7] C. J. Simpson, C. E. Ells, Delayed hydrogen embrittlement in Zr-2.5 wt\% Nb, J. Nucl. Mater. 52:2 (1974) 289-295.

[8] H. M. Chung, R. S. Daum, J. M. Hiller, M. C. Billone, Characteristics of Hydride Precipitation and Reorientation in Spent-Fuel Cladding, Zirconium in the Nuclear Industry: Thirteenth International Symposium, ASTM STP 1423, G. D. Moan, P. Rudling, Eds., ASTM International (2002) 561582

[9] G. Carpenter, The precipitation of $\gamma$-zirconium hydride in zirconium, Acta Metall. 26 (1978) 1225-1235.

[10] G. C. Weatherly, The precipitation of $\gamma$-hydride plates in zirconium, Acta Metall. 29:3 (1981) 501-512.

[11] V. Perovic, G. C. Weatherly, C. J. Simpson, Hydride precipitation in $\alpha / \beta$ zirconium alloys, Acta Metall. 31:9 (1983) 1381-1391.

[12] J. S. Bradbrook, G. W. Lorimer, N. Ridley, The precipitation of zirconium hydride in zirconium and zircaloy-2, J. Nucl. Mater. 42:2 (1972) 142-160.

[13] J. E. Bailey, Electron microscope observations on the precipitation of zirconium hydride in zirconium, Acta Metall. 11:4 (1963) 267-280. 
[14] G. J. C. Carpenter, J. F. Watters, R. W. Gilbert, Dislocations generated by zirconium hydride precipitates in zirconium and some of its alloys, J. Nucl. Mater. 48:3 (1973) 267-276.

[15] R. Maaß, S. Van Petegem, D. Ma, J. Zimmermann, D. Grolimund, F. Roters, H. Van Swygenhoven, D. Raabe, Smaller is stronger: The effect of strain hardening, Acta Mater. 57:20 (2009) 5996-6005.

[16] R. Maaß, S. Van Petegem, H. Van Swygenhoven, P. Derlet, C. Volkert, D. Grolimund, Time-Resolved Laue Diffraction of Deforming Micropillars, Phys. Rev. Lett. 99:14 (2007) 145505.

[17] R. Maa $\beta$, D. Grolimund, S. Van Petegem, M. Willimann, M. Jensen, H. Van Swygenhoven, T. Lehnert, M. A. M. Gijs, C. A. Volkert, E. T. Lilleodden, R. Schwaiger, Defect structure in micropillars using x-ray microdiffraction, Appl. Phys. Lett. 89:15 (2006) 151905.

[18] B. A. Cheadle, C. E. Ells, W. Evans, The development of texture in zirconium alloy tubes, J. Nucl. Mater. 23:2 (1967) 199-208.

[19] G. Bertolino, G. Meyer, J. Perez Ipina, Degradation of the mechanical properties of Zircaloy-4 due to hydrogen embrittlement, J. Alloys and Compd. (2001) 408-413.

[20] R. Barabash, G. E. Ice, B. C. Larson, G. M. Pharr, K. S. Chung, W. Yang, White microbeam diffraction from distorted crystals, Appl. Phys. Lett. 79:6 (2001) 749-751.

[21] R. Maaß, M. D. Uchic, In-situ characterization of the dislocation-structure evolution in Ni micro-pillars, Acta Mater. 60:3 (2012) 1027-1037.

[22] J. Zimmermann, S. Van Petegem, H. Bei, D. Grolimund, Effects of focused ion beam milling and pre-straining on the microstructure of directionally solidified molybdenum pillars: A Laue diffraction analysis, Scr. Mater. 62 (2010) 746-749

[23] R. Maaß, S. Van Petegem, J. Zimmermann, C. N. Borca, On the initial microstructure of metallic micropillars, Scr. Mater. 59 (2008) 471-474

[24] R. Maaß, S. Van Petegem, C. N. Borca, H. Van Swygenhoven, In situ Laue diffraction of metallic micropillars, Mat. Sci. Eng. A 524:1-2 (2009) 40-45.

[25] G. E. Ice, R. I. Barabash, White Beam Micro diffraction and Dislocations Gradients, in: Dislocations in Solids, Elsevier (2007) 499-601.

[26] C. R. Hutchinson, R. E. Hackenberg, G. J. Shiflet, A comparison of EDS microanalysis in FIB-prepared and electropolished TEM thin foils, Ultramicroscopy. 94:1 (2003) 37-48. 
[27] D. Kiener, C. Motz, G. Dehm, Dislocation-induced crystal rotations in micro-compressed single crystal copper columns, J. Mater. Sci. $43: 7$ (2008) 2503-2506.

[28] L. Wang, Y. Yang, P. Eisenlohr, T. R. Bieler, M. A. Crimp, D. E. Mason, Twin Nucleation by Slip Transfer across Grain Boundaries in Commercial Purity Titanium, Metall. Trans. A 41:2 (2009) 421-430.

[29] R. I. Barabash, G. E. Ice, F. J. Walker, Quantitative microdiffraction from deformed crystals with unpaired dislocations and dislocation walls, J. Appl. Phys. 93:3 (2003) 1457-1464.

[30] N. A. Fleck, G. M. Muller, M. F. Ashby, J. W. Hutchinson, Strain gradient plasticity: theory and experiment, Acta. Metall. Mater. 42:2 (1994) 475487.

[31] L. A. Simpson, C. D. Cann, Fracture toughness of zirconium hydride and its influence on the crack resistance of zirconium alloys, J. Nucl. Mater. 87 (1979) 303-316.

[32] C. E. Coleman, D. Hardie, The hydrogen embrittlement of $\alpha$-zirconium-A review, J. Less-Common Met. 11 (1966) 168-185.

[33] K. G. Barraclough, C. J. Beevers, Some observations on the deformation characteristics of bulk polycrystalline zirconium hydrides, J. Mater. Sci. 4:6 (1969) 518-525.

[34] C. J. Beevers, K. G. Barraclough, Some observations on the deformation characteristics of bulk polycrystalline zirconium hydrides, J. Mater. Sci. 4:9 (1969) 802-808.

[35] A. Barrow, A. Korinek, M. R. Daymond, Evaluating zirconium-zirconium hydride interfacial strains by nano-beam electron diffraction, J. Nucl. Mater. 432 (2013) 366-370.

[36] G. Carpenter, The dilatational misfit of zirconium hydrides precipitated in zirconium, J. Nucl. Mater. 48 (1973) 264-266. 


\section{List of Figures}

1 (a) Hydride habit planes in $\alpha$-Zr: $\{10 \overline{1} 7\}$ for macroscopic hydride packets and $\{0002\}$ for microscopic hydride platelets (illustration adapted from [8], (b) Dislocations generated by the nucleation of hydride precipitates in the $\mathrm{Zr}$ matrix (illustration adapted from [14], (c-d) Optical micrographs of electrolyically hydrided, hot-rolled and recrystallised Zircaloy-4 before (c) and after (d) annealing heat treatment to promote both grain growth and intragranular hydride precipitation. . . . . . . . . . .

2 (a) Secondary electron micrograph of surface trench fabricated through perpendicular FIB milling in order to observe hydride orientation with respect to loading axis. Location of subsequent pillar with respect to hydride is outlined, (b-c) Simplified schematics of loading rigs used during in situ micropillar compression: (b) in situ SEM set-up: Alemnis SEM indenter fitted within an SEM chamber, (c) in situ diffraction set-up with a customised nano-indentation loading rig incorporating a Hysitron indenter. . 21

3 Load-displacement curves (with inferred secondary stress axis) obtained from in situ SEM compressive loading of both as-received (AR1) and hydride-containing (H1, H2) micropillars. The yield points are identified. Micrographs corresponding to points (a)-(c) in each loading curve are shown in Figures 4,6 and 7. . . . . 22

4 Evolution in the appearance of the as-received pillar sample during loading, at the points identified in Figure 3. Shortly after yielding, a shear band can be identified, followed by the appearance of a second shear band which is present only in the lower section of the pillar. . . . . . . . . . . . . . 23

5 FIB-milled surface of the unhydrided sample AR1 after deformation. (a) SE micrograph of the sample surface, showing the location of the slip band, (b) outline of the pillar encased in the protective Pt deposit, (c) EBSD map of the pillar (IPF coloured) and (d) orientations of the material above and below the shear band. . . . . . . . . . . . . . . . . .

6 Evolution of hydrided sample H1 during deformation (a)-(c), plus observation of the sample after loading with improved resolution (d). The location of hydride packets (1) and (2) are outlined. The red circles indicate the first (b) and second (c) shear bands to form, (e)-(f) SE images of each side of the hydride packet after compression showing evidence of slip between the individual stacked platelets (pillar orientation: $[0001]_{\alpha} \sim 30^{\circ}$ to loading axis). 25

$7 \quad$ Evolution of hydrided sample H2 during deformation (a)-(c), and observation of the sample after loading with improved resolution (d). In (d), the hydride packets are outlined; red circles indicate the first (b) and second (c) shear bands to form (pillar orientation: $[0001]_{\alpha} \sim 55^{\circ}$ to loading axis). . . . . . . . . 26 
8 FIB-milled surface analysis of the hydrided sample, H2, after deformation. (a) SE micrograph of the sample surface highlighting the location of the hydride packets and the location of the corresponding EBSD map, (b) EBSD map of the pillar (IPF coloured), (c) Misorientation profiles, from a reference point at the beginning of each line profile, across three regions within the hydride packets, (d) Orientations of the hydride at locations (1) and (2), represented by green and black crosses respectively. . . . . . . . 27

9 Load-displacement curves (with inferred secondary stress axis) obtained from in situ diffraction compressive loading of both asreceived (ARL1) and hydride containing (HL1, HL2) micropillars. For ARL1, the Laue diffraction patterns corresponding to points $\mathrm{b}_{1}-\mathrm{b}_{4}$ are shown in Figure 15, whilst points $1-8$ on the loading curves for HL1-HL2 refer to the diffraction patterns in Figure 16. 28

10 Secondary electron images of the as-received pillar, ARL1 (a), and both hydride-containing pillars - HL1 (b) and HL2 (c). (1) refers to before compression and (2) refers to after. The locations of the hydride packets before deformation in pillars HL1 and HL2 are shown. . . . . . . . . . . . . . . . . . 29

11 Indexing of experimental diffraction spots by comparison with simulated Laue diffraction patterns. (a) experimental diffraction pattern from hydride-containing pillar HL1. (b) simulated pattern developed using the crystal orientation of the sample obtained from EBSD. Both intensity scales are presented in terms of scattering factors (SF), and the origin corresponds to the position of the undiffracted (straight-through) beam. . . . . . . 30

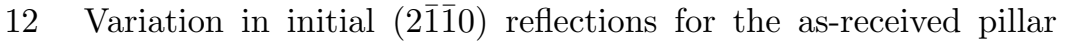
ARL1 (a), and both hydride-containing pillars HL1, HL2 (b-c), before loading. Distinct maxima are highlighted in (b-c). (d-f) provide a hypothesis are to the source of the distinct maxima from differing locations in the gauge volume (orange) in each pillar. The white arrows point towards the origin of each pattern. 31

13 Simulated diffraction patterns (a) for the two phases and (b) effect of increases in the $\alpha$-Zr axial ratio $(c / a)$. The $\mathrm{ZrH}$ spots are in red and the $\mathrm{Zr}$ spots in grey. The direction and size of the arrows indicates the effect of increases in $c / a \ldots \ldots . . . . .32$

14 (a-c) Effect of crystal rotations and (d-f) effect of $\left(10^{\circ}\right)$ shears of the unit cell on the simulated Laue diffraction patterns. The colour of the spots indicates their intensity, the dotted box the outline of the detector and the magnitude and direction of the arrows indicates the effect on the peaks. The $((2 \overline{1} \overline{1} 0)$ and $(\overline{1} 2 \overline{1} 0)$ reflections are highlighted. . . . . . . . . . . . . 33 
15 In situ compression of the unhydrided pillar, AL1. $\left(\mathrm{a}_{1}\right)$ shows the pillar after deformation and outlines the location of the EBSD map presented in $\left(\mathrm{a}_{2}\right) \cdot\left(\mathrm{c}_{1}-\mathrm{c}_{2}\right)$ show the misorientation profiles across lines (1) and (2) labelled in $\left(\mathrm{a}_{2}\right)$. $\left(\mathrm{b}_{1}-\mathrm{b}_{4}\right)$ track the evolution of $(2 \overline{1} \overline{1} 0)$ peak with increasing load, at the points identified in Figure 9 (the white arrows point towards the origin of each pattern) and (d) shows the expected effect of twinning on the corresponding diffraction pattern; $\left(\mathrm{d}_{\mathrm{inset}}\right)$ illustrates the orientation of both parent and twin crystals. . . . . . . . . . . .

16 Evolution of diffraction peaks for both hydride containing samples, HL1 and HL2, during the applied load cycle. (a),(c) track the $(2 \overline{1} 10)$ peak for HL1 and HL2 respectively. (b) tracks the (1120) peak for HL1 (peak not visible for HL2). The number on each 2D plot corresponds to the equivalent point in Figure 9 and the white arrows point towards the origin of each pattern. . . . 35

17 (a) Hydride-containing pillar H1, deformed in the SEM, with box highlighting the location of the TEM foil removed by FIB sectioning. (b) diffraction pattern from the hydride packet (red circles highlight peak streaking), (c, d) low magnification views of the pillar in bright and dark field $(\mathrm{BF} / \mathrm{DF})$ imaging . . . . . . . . .

18 (a) Hydride containing pillar H2, deformed in the SEM, showing location of the TEM foil removed. (b-d) low magnification view of the TEM foil, showing several distinct hydride platelets. (e) High resolution phase contrast image of a $\mathrm{ZrH}$ packet-packet interface, and (f) diffraction patterns from the $\mathrm{ZrH}$ packet-packet interface (red circles highlight peak streaking). . . . . . . . . . . . 37

19 (a) TEM foil removed from hydride-containing in situ X-ray Laue diffraction pillar HL1. (b) Diffraction pattern from the centre of the pillar region imaged (boxed), showing the hydride and twin. (c-d) High resolution phase contrast imaging of defects within the hydride platelet, at the parent/twin interface (P: Parent, T: Twin). 38 

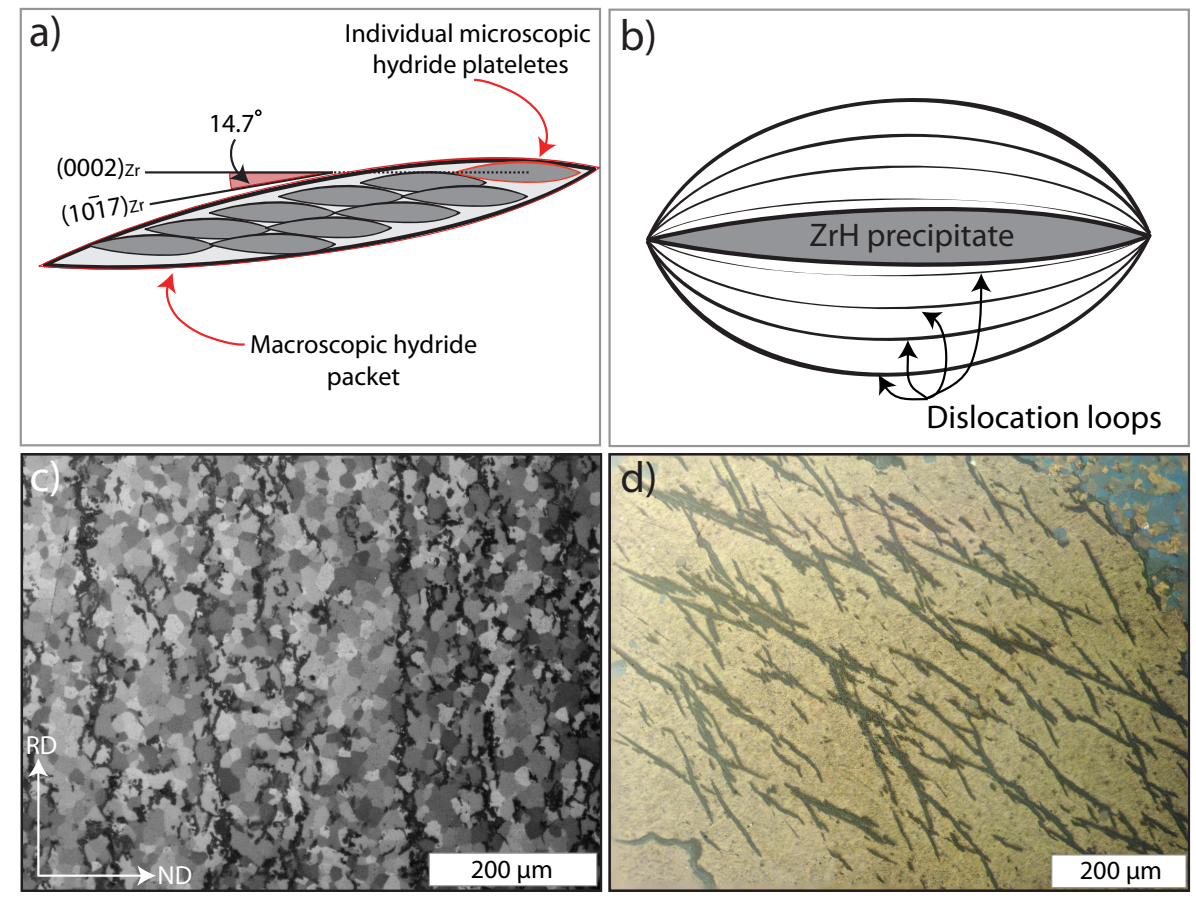

Figure 1: (a) Hydride habit planes in $\alpha$-Zr: $\{10 \overline{1} 7\}$ for macroscopic hydride packets and $\{0002\}$ for microscopic hydride platelets (illustration adapted from [8], (b) Dislocations generated by the nucleation of hydride precipitates in the $\mathrm{Zr}$ matrix (illustration adapted from [14], (c-d) Optical micrographs of electrolyically hydrided, hot-rolled and recrystallised Zircaloy- 4 before (c) and after (d) annealing heat treatment to promote both grain growth and intragranular hydride precipitation. 

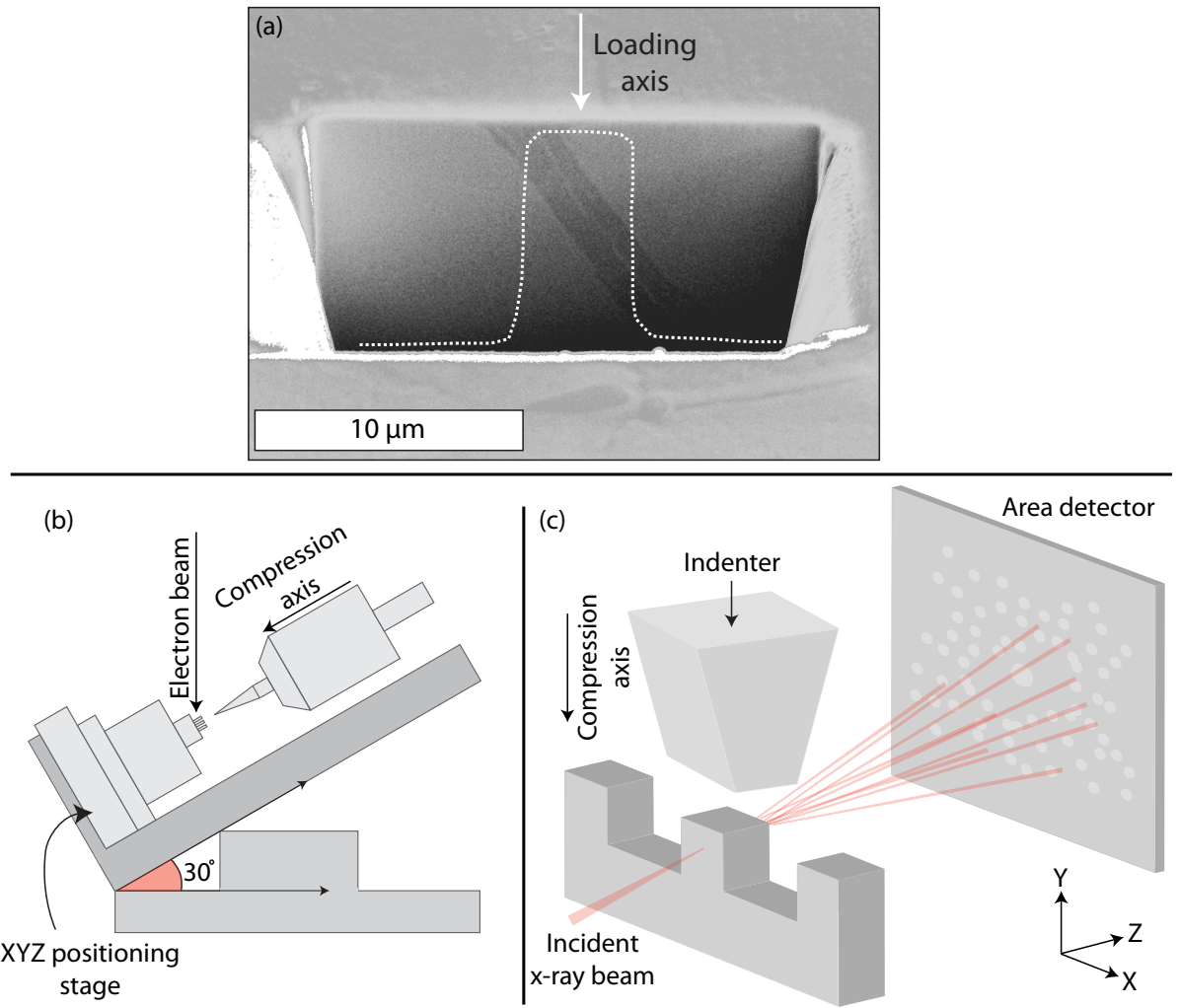

Figure 2: (a) Secondary electron micrograph of surface trench fabricated through perpendicular FIB milling in order to observe hydride orientation with respect to loading axis. Location of subsequent pillar with respect to hydride is outlined, (b-c) Simplified schematics of loading rigs used during in situ micropillar compression: (b) in situ SEM set-up: Alemnis SEM indenter fitted within an SEM chamber, (c) in situ diffraction set-up with a customised nanoindentation loading rig incorporating a Hysitron indenter. 

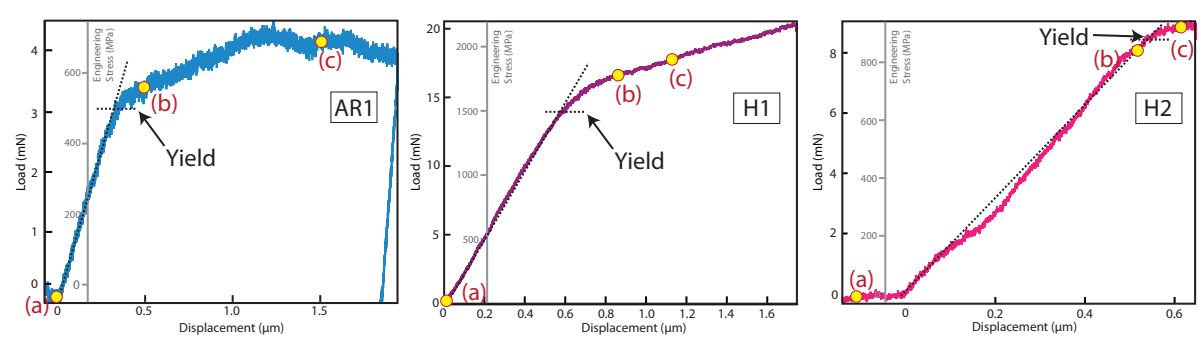

Figure 3: Load-displacement curves (with inferred secondary stress axis) obtained from in situ SEM compressive loading of both as-received (AR1) and hydride-containing (H1, H2) micropillars. The yield points are identified. Micrographs corresponding to points (a)-(c) in each loading curve are shown in Figures 4, 6 and 7 . 

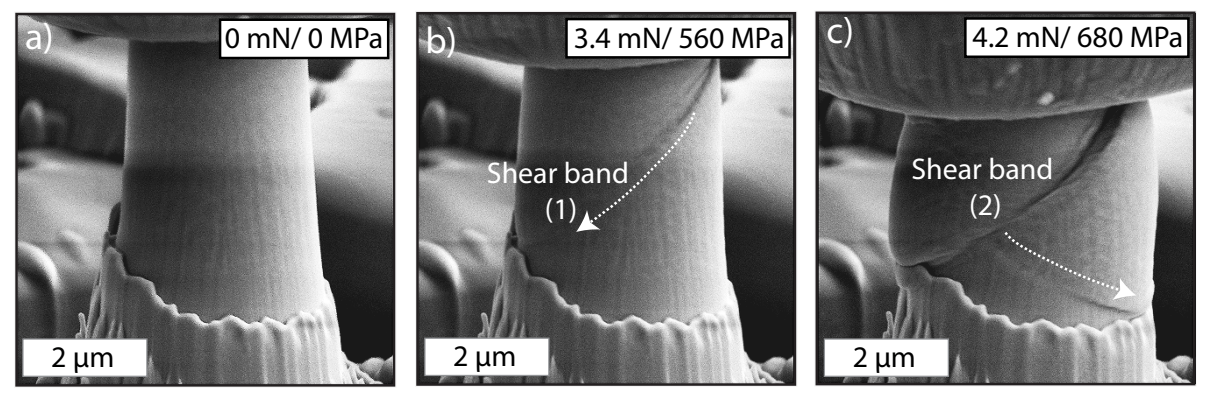

Figure 4: Evolution in the appearance of the as-received pillar sample during loading, at the points identified in Figure 3. Shortly after yielding, a shear band can be identified, followed by the appearance of a second shear band which is present only in the lower section of the pillar. 


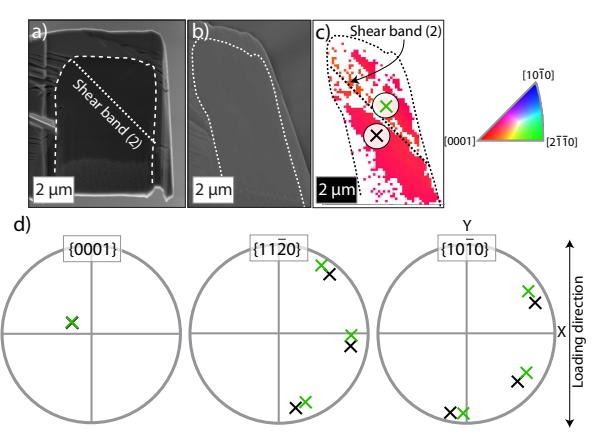

Figure 5: FIB-milled surface of the unhydrided sample AR1 after deformation. (a) SE micrograph of the sample surface, showing the location of the slip band, (b) outline of the pillar encased in the protective Pt deposit, (c) EBSD map of the pillar (IPF coloured) and (d) orientations of the material above and below the shear band. 


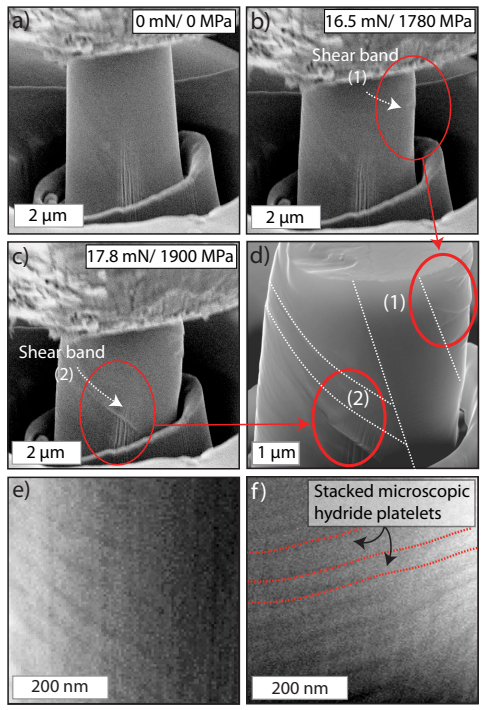

Figure 6: Evolution of hydrided sample H1 during deformation (a)-(c), plus observation of the sample after loading with improved resolution (d). The location of hydride packets (1) and (2) are outlined. The red circles indicate the first (b) and second (c) shear bands to form, (e)-(f) SE images of each side of the hydride packet after compression showing evidence of slip between the individual stacked platelets (pillar orientation: $[0001]_{\alpha} \sim 30^{\circ}$ to loading axis). 


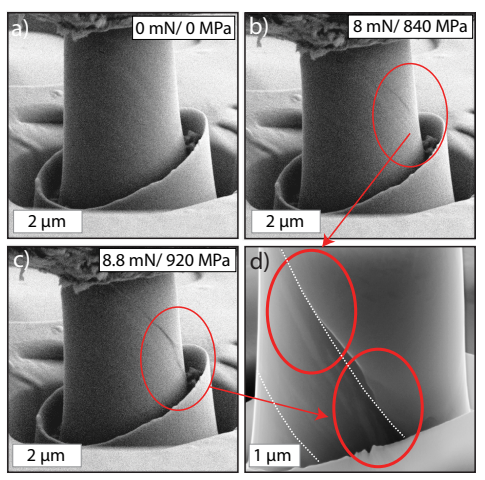

Figure 7: Evolution of hydrided sample $\mathrm{H} 2$ during deformation (a)-(c), and observation of the sample after loading with improved resolution (d). In (d), the hydride packets are outlined; red circles indicate the first (b) and second (c) shear bands to form (pillar orientation: $[0001]_{\alpha}$ $\sim 55^{\circ}$ to loading axis). 

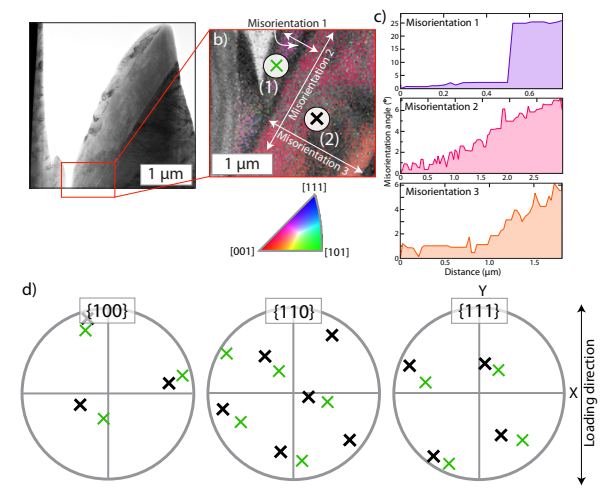

Figure 8: FIB-milled surface analysis of the hydrided sample, H2, after deformation. (a) SE micrograph of the sample surface highlighting the location of the hydride packets and the location of the corresponding EBSD map, (b) EBSD map of the pillar (IPF coloured), (c) Misorientation profiles, from a reference point at the beginning of each line profile, across three regions within the hydride packets, (d) Orientations of the hydride at locations (1) and (2), represented by green and black crosses respectively. 

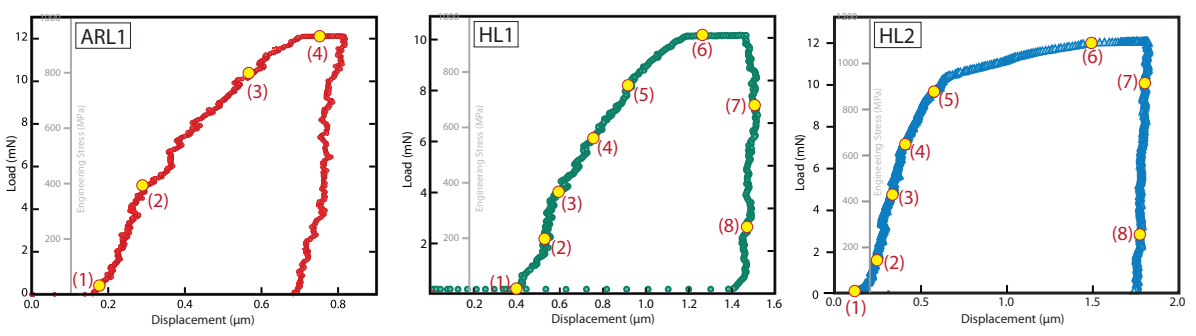

Figure 9: Load-displacement curves (with inferred secondary stress axis) obtained from in situ diffraction compressive loading of both as-received (ARL1) and hydride containing (HL1, HL2) micropillars. For ARL1, the Laue diffraction patterns corresponding to points $\mathrm{b}_{1}-\mathrm{b}_{4}$ are shown in Figure 15, whilst points 1-8 on the loading curves for HL1-HL2 refer to the diffraction patterns in Figure 16. 


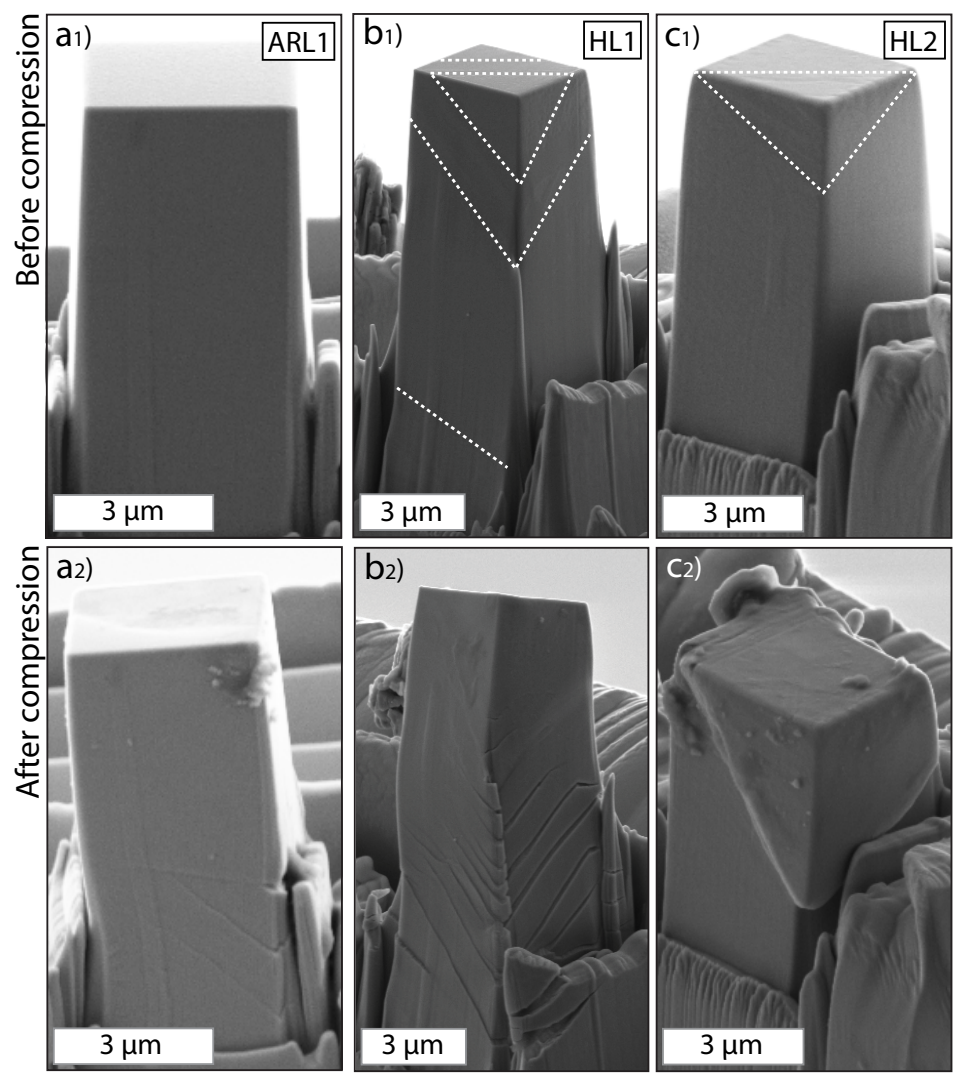

Figure 10: Secondary electron images of the as-received pillar, ARL1 (a), and both hydridecontaining pillars - HL1 (b) and HL2 (c). (1) refers to before compression and (2) refers to after. The locations of the hydride packets before deformation in pillars HL1 and HL2 are shown. 


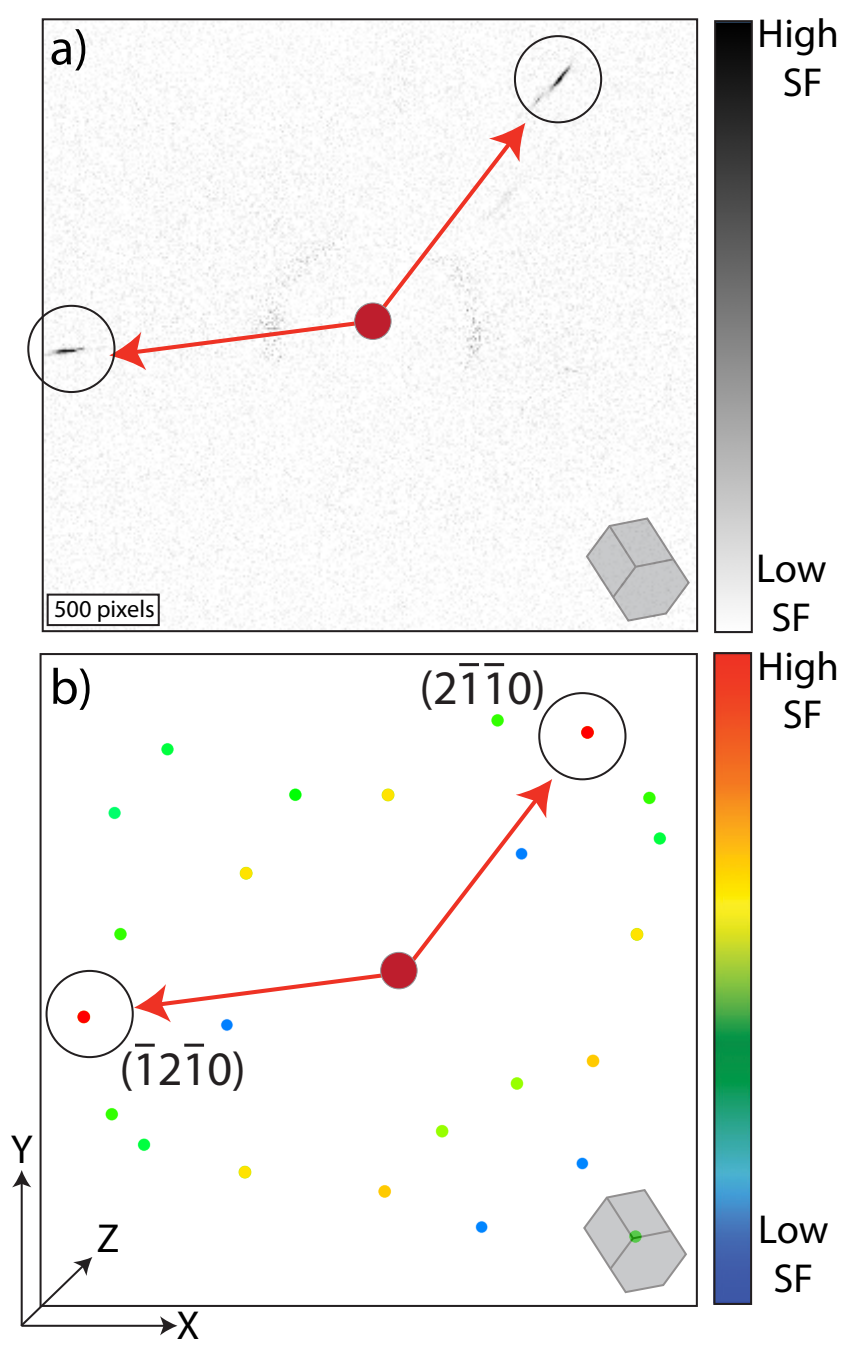

Figure 11: Indexing of experimental diffraction spots by comparison with simulated Laue diffraction patterns. (a) experimental diffraction pattern from hydride-containing pillar HL1. (b) simulated pattern developed using the crystal orientation of the sample obtained from EBSD. Both intensity scales are presented in terms of scattering factors (SF), and the origin corresponds to the position of the undiffracted (straight-through) beam. 


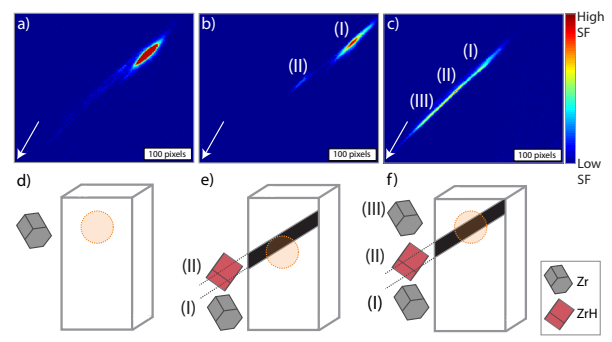

Figure 12: Variation in initial (21̄10) reflections for the as-received pillar ARL1 (a), and both hydride-containing pillars HL1, HL2 (b-c), before loading. Distinct maxima are highlighted in (b-c). (d-f) provide a hypothesis are to the source of the distinct maxima from differing locations in the gauge volume (orange) in each pillar. The white arrows point towards the origin of each pattern. 


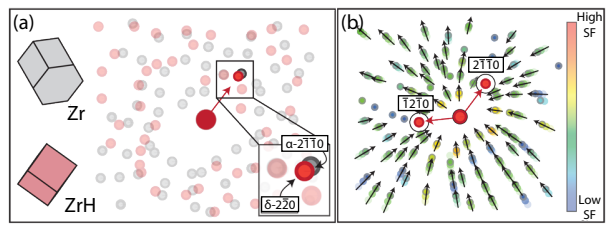

Figure 13: Simulated diffraction patterns (a) for the two phases and (b) effect of increases in the $\alpha$ - $\mathrm{Zr}$ axial ratio $(c / a)$. The $\mathrm{ZrH}$ spots are in red and the $\mathrm{Zr}$ spots in grey. The direction and size of the arrows indicates the effect of increases in $c / a$. 

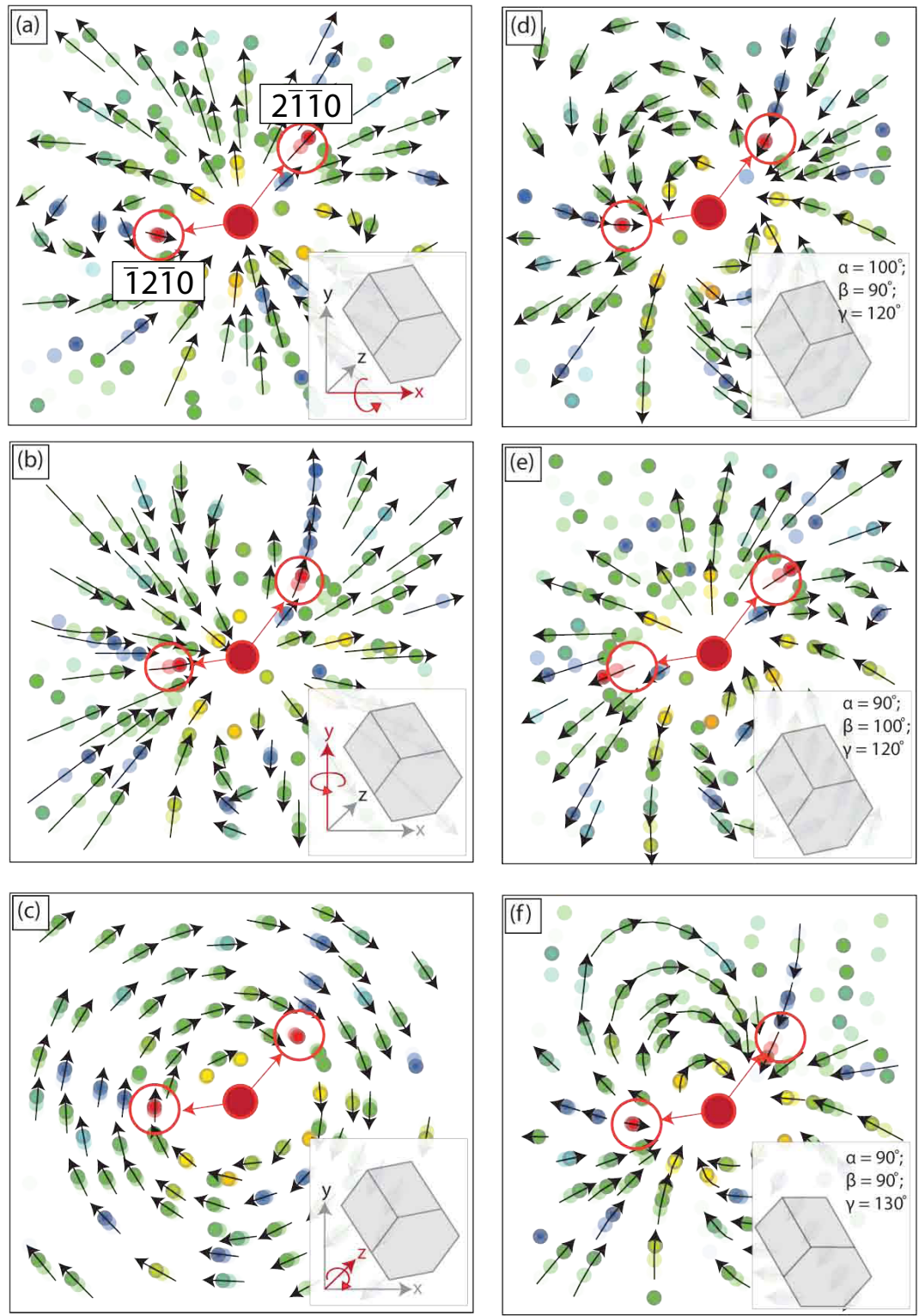

Figure 14: (a-c) Effect of crystal rotations and (d-f) effect of $\left(10^{\circ}\right)$ shears of the unit cell on the simulated Laue diffraction patterns. The colour of the spots indicates their intensity, the dotted box the outline of the detector and the magnitude and direction of the arrows indicates the effect on the peaks. The $((2 \overline{1} \overline{1} 0)$ and $(\overline{1} 2 \overline{1} 0)$ reflections are highlighted. 


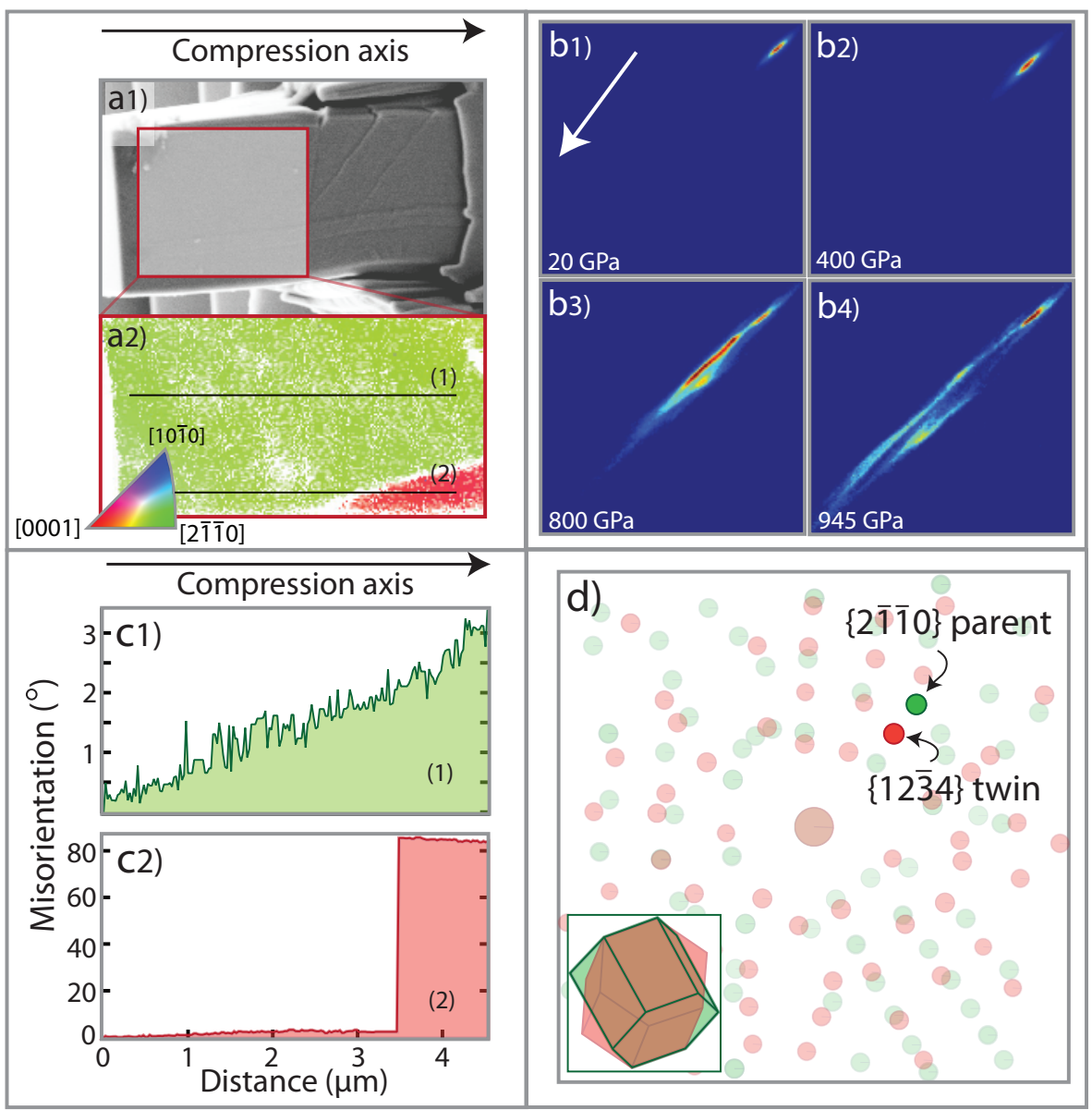

Figure 15: In situ compression of the unhydrided pillar, AL1. ( $\left.\mathrm{a}_{1}\right)$ shows the pillar after deformation and outlines the location of the EBSD map presented in $\left(a_{2}\right) .\left(c_{1}-c_{2}\right)$ show the misorientation profiles across lines (1) and (2) labelled in $\left(\mathrm{a}_{2}\right)$. ( $\left.\mathrm{b}_{1}-\mathrm{b}_{4}\right)$ track the evolution of $(2 \overline{1} \overline{1} 0)$ peak with increasing load, at the points identified in Figure 9 (the white arrows point towards the origin of each pattern) and (d) shows the expected effect of twinning on the corresponding diffraction pattern; $\left(\mathrm{d}_{\mathrm{inset}}\right)$ illustrates the orientation of both parent and twin crystals. 


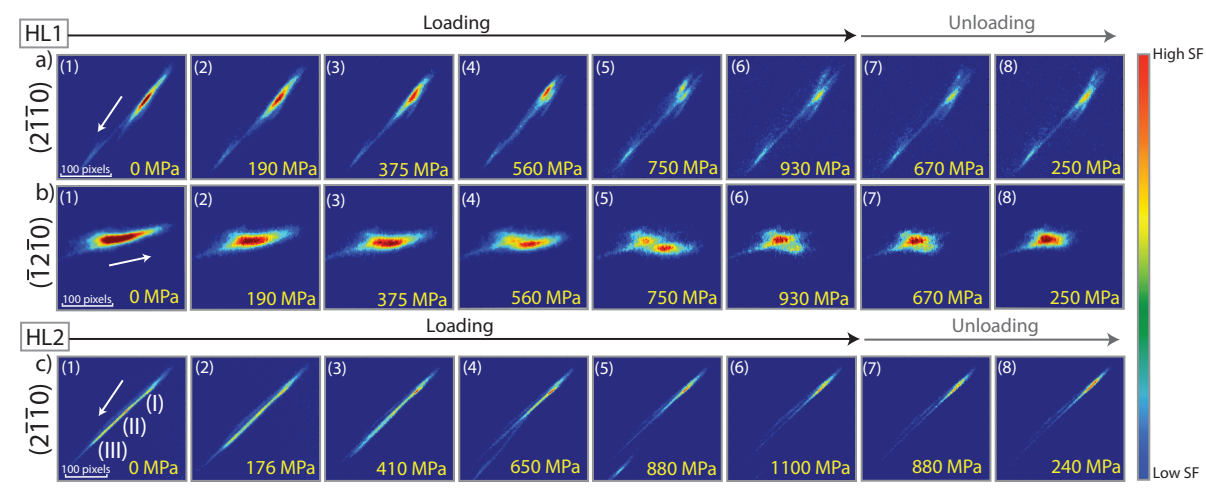

Figure 16: Evolution of diffraction peaks for both hydride containing samples, HL1 and HL2,

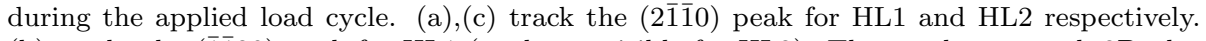
(b) tracks the $(\overline{1} \overline{1} 20)$ peak for HL1 (peak not visible for HL2). The number on each $2 \mathrm{D}$ plot corresponds to the equivalent point in Figure 9 and the white arrows point towards the origin of each pattern. 


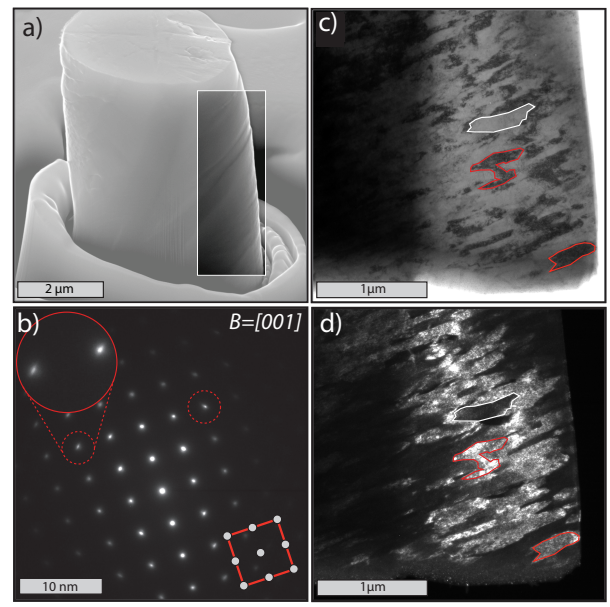

Figure 17: (a) Hydride-containing pillar H1, deformed in the SEM, with box highlighting the location of the TEM foil removed by FIB sectioning. (b) diffraction pattern from the hydride packet (red circles highlight peak streaking), (c, d) low magnification views of the pillar in bright and dark field (BF/DF) imaging 


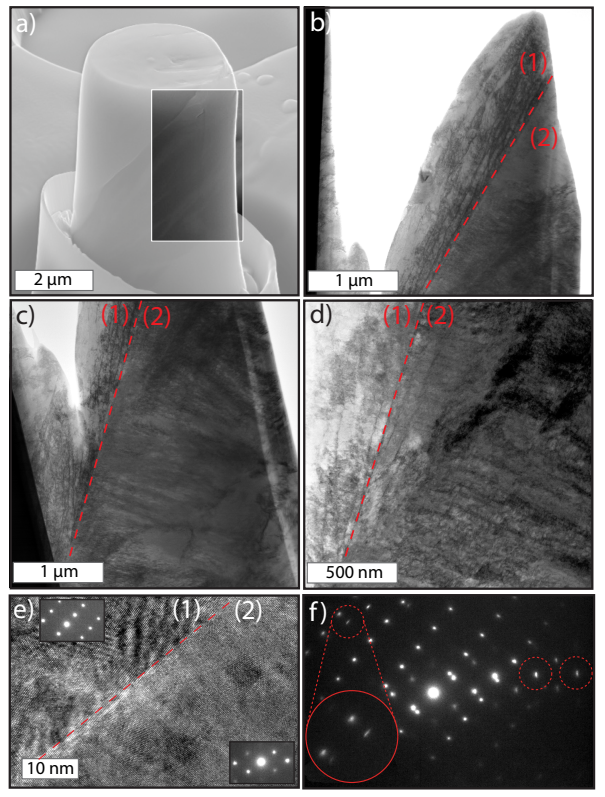

Figure 18: (a) Hydride containing pillar H2, deformed in the SEM, showing location of the TEM foil removed. (b-d) low magnification view of the TEM foil, showing several distinct hydride platelets. (e) High resolution phase contrast image of a $\mathrm{ZrH}$ packet-packet interface, and (f) diffraction patterns from the $\mathrm{ZrH}$ packet-packet interface (red circles highlight peak streaking). 


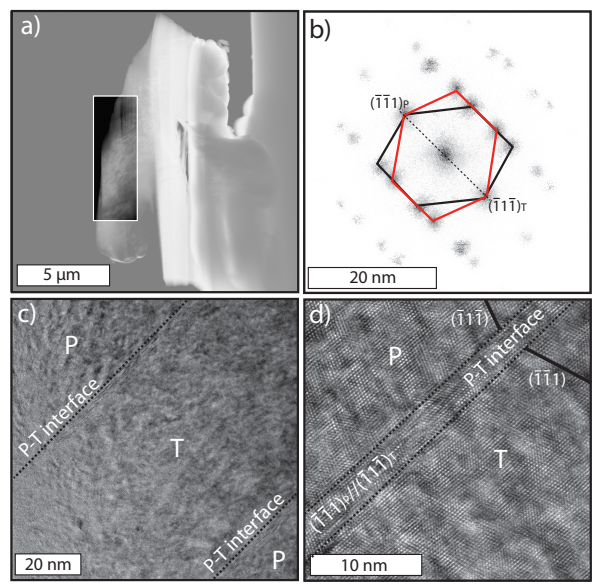

Figure 19: (a) TEM foil removed from hydride-containing in situ X-ray Laue diffraction pillar HL1. (b) Diffraction pattern from the centre of the pillar region imaged (boxed), showing the hydride and twin. (c-d) High resolution phase contrast imaging of defects within the hydride platelet, at the parent/twin interface (P: Parent, T: Twin). 